\title{
The most optimal device for removing space debris
}

\author{
Bin Ren ${ }^{1, a}$ \\ ${ }^{1}$ School of North China Electric Power University, Baoding 071003, China; \\ a1624352537@qq.com
}

Keywords: hierarchical structure model, structure the comparison matrix, total sorts of the layers

\begin{abstract}
According to the data we have consulted, we can come to the conclusion that there are five kinds of commonly used methods to get rid of space junk, respectively Laser Removal Technology, Satellite Debris Collector, Space Garbage Truck, Suicide Satellite and Resistance-increase Device. Their respective advantages and disadvantages are different, in this paper, through the establishment of the AHP model, we analyze the different combinations, and get the conclusion of the top three. These schemes can make automatic space debris removal device having a better use value and market competitiveness.
\end{abstract}

\section{Introduction}

A lot of ways to sweep up space debris exist now, however some methods are in theory. Based on their working principles, we classify and select five kinds of typical schemes.

a. Laser Removal Technology

The working principle of laser removal technology roughly can be divided into two kinds. The first is that a laser can make the pieces burning and fusing, or give thrust to the debris, and using a laser irradiation can make debris' temperature go up until sublimation. The second is that the laser beam to the surface of debris, and produce certain thrust, making the debris go into the atmosphere or garbage track down rail. Debris size applicable to the technology is less than $0.1 \mathrm{~m}$, and according to different pieces of track, the strategy is different. Its advantage is repeatedly used, high efficiency; while high power laser devices and target tracking accuracy are the main technical difficulties.

b. Satellite Debris Collector

A large-area debris collector can capture lots of small pieces on LEO, the removing effect is obvious, the size of debris less than $0.1 \mathrm{~m}$ can be applicable to the technology. Its advantage is high efficiency. But the device must have a huge area and can spread in orbit, and it must spend a lot of time to clear the debris.

c. Space Garbage Truck

Space garbage truck will collect and encapsulate large pieces of space debris placed in the specified orbit and then shipped them to orbit nearer to the earth. The vehicle can also collect the whole piece of old rockets residues .But the problem is its high cost and complicated operation.

d. Suicide Satellite

The volume of a suicide satellite is as small as football. When detected the space junk, the suicide satellite will attach itself on the garbage, reduce its speed and finally get into the atmosphere, ending up with space junk. This method advantages in low cost, strong ability of survival and calculation.

e. Resistance-increase Device

Attaching this kind of devices such as inflatable balloon or thin film sail on debris will increase its resistance caused by atmospheric drag, lower the debris track quickly and shorten orbit life .The applicable size of debris is greater than $1 \mathrm{~m}$. The technology takes advantage in its high efficiency, low cost but faces risk of collision with other spacecraft or debris in the process of lowering the debris track which is caused by the large area of the device. 


\section{AHP Model}

STEP 1 Establishing a hierarchical structure model of the combination plan

The purpose of establishing the model is preliminary selecting several suitable combinations. According to what we have collected, we combine every two of the five cleaning ways, and write as follows,

Table 1.Combinations plan

\begin{tabular}{cc|cc}
\hline Definition & Combinations & Definition & Combinations \\
\hline$C_{1}$ & $L R T+S D C$ & $C_{6}$ & $S D C+S S$ \\
$C_{2}$ & $L R T+S G T$ & $C_{7}$ & $S D C+R I D$ \\
$C_{3}$ & $L R T+S S$ & $C_{8}$ & $S G T+S S$ \\
$C_{4}$ & $L R T+R I D$ & $C_{9}$ & $S G T+S S$ \\
$C_{5}$ & $S D C+S G T$ & $C_{10}$ & $S S+R I D$ \\
\hline
\end{tabular}

Which $L R T$ refers to the Laser removal technology, SDC refers to Debris collector, SGT stands for Space garbage truck, SS represents Suicide satellite, and RID means increasing resistance device.

Each independent alternative is applicable to space debris of different sizes and the money needed is not the same. According to the analysis of data, we identify three principles, namely the ability to clean up space debris, the estimated price, and the removal efficiency. Then they are applied to evaluate and rank the combinations.

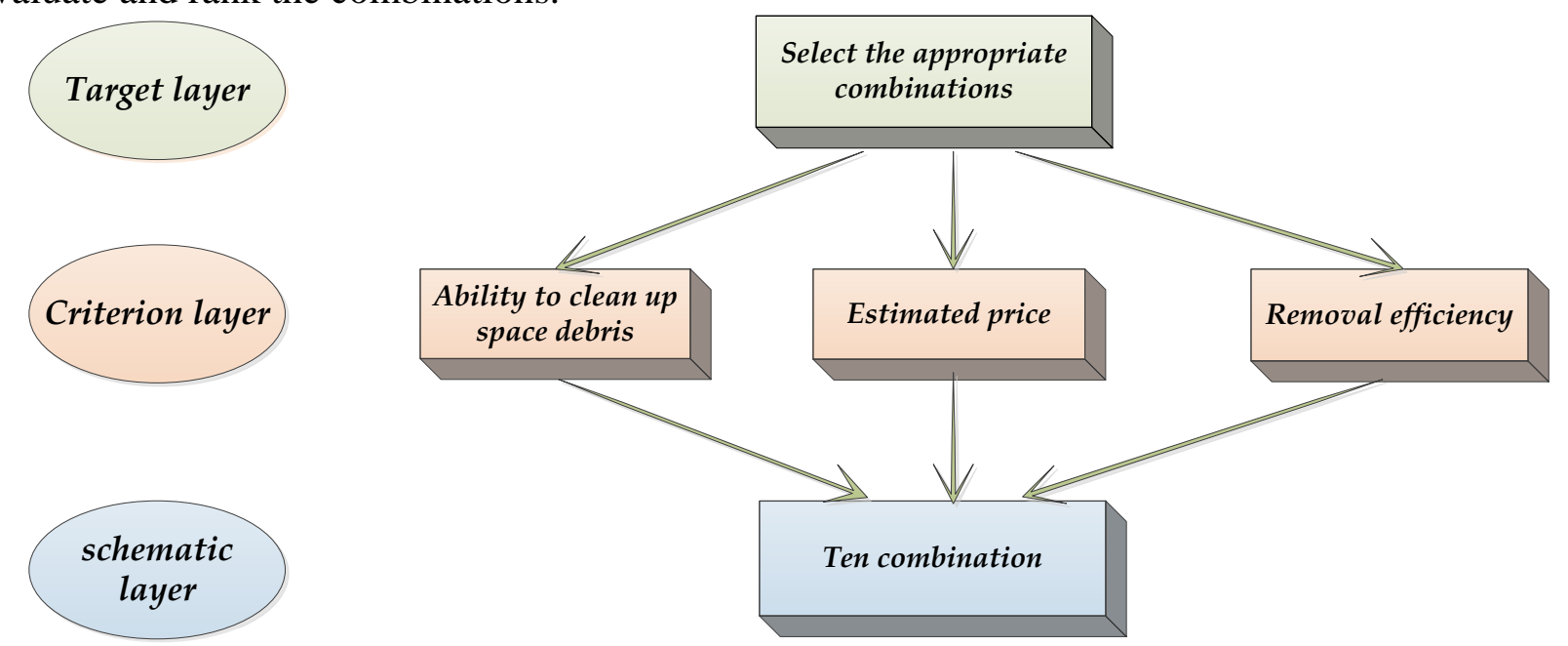

Figure 1.The flow chart of AHP model

STEP 2 Structure the comparison matrix and the single-sort The paired comparison of the matrix rule layer is

$$
A=\left[\begin{array}{ccc}
1 & 2 & 1 / 3 \\
1 / 2 & 1 & 1 / 5 \\
3 & 5 & 5
\end{array}\right]
$$

From calculating, we can consult that the largest eigenvalue of $A, \lambda_{\max }=3.0037$. The corresponding eigenvectors, $W^{(2)}=\left[\begin{array}{l}0.32880 .17470 .9281\end{array}\right]^{\prime}$, consistency ratio is $C R=0.0032$. 


$$
B_{1}^{(10)}=\left[\begin{array}{cccccccccr}
1 & 1 / 6 & 1 / 7 & 1 / 5 & 1 / 6 & 1 / 7 & 1 / 5 & 1 / 3 & 1 / 2 & 1 / 3 \\
6 & 1 & 1 / 3 & 2 & 1 & 1 & 2 & 3 & 3 & 2 \\
7 & 3 & 1 & 2 & 1 / 3 & 1 & 2 & 2 & 4 & 2 \\
5 & 1 / 2 & 1 / 2 & 1 & 1 / 2 & 1 / 3 & 1 & 1 & 3 & 2 \\
6 & 1 & 3 & 2 & 1 & 1 / 2 & 2 & 2 & 3 & 3 \\
7 & 1 & 1 & 3 & 2 & 1 & 3 & 2 & 6 & 3 \\
5 & 1 / 2 & 1 / 2 & 1 & 1 / 2 & 1 / 3 & 1 & 2 & 3 & 1 / 5 \\
3 & 1 / 3 & 1 / 2 & 1 & 1 / 2 & 1 / 2 & 1 / 2 & 1 & 2 & 2 \\
2 & 1 / 3 & 1 / 4 & 1 / 3 & 1 / 3 & 1 / 6 & 1 / 3 & 1 / 2 & 1 & 1 / 4 \\
3 & 1 / 2 & 1 / 2 & 1 / 2 & 1 / 3 & 1 / 3 & 5 & 1 / 2 & 4 & 1
\end{array}\right]
$$

$B_{1}^{(10)}$ is a pairwise comparison matrix of space debris' processing capacity, which is compared according to the range of debris cleaning. For example, $C_{1}$ can only cleaning the pieces that is less than $0.1 \mathrm{~m}$ while $C_{2}$ can clean up both the pieces less than $0.1 \mathrm{~m}$ and more than $1 \mathrm{~m}$, who's clearing range is wider than $C_{1} \cdot B_{1}^{(10)}(2,1)$ is relatively larger when this indicator reflected on the pairwise comparison matrix .So we can work out ten different combinations of the pairwise comparison matrix according to the rule of ability to clean up space debris.

By the same token, we can give the pairwise comparison matrix of price estimation and the removal efficiency. Because the matrix is too large, we list $B_{2}^{(10)}$ and $B_{3}^{(10)}$ in the appendix, and we can get Table 2 from these matrices.

Table 2.The maximum characteristic value of each attribute

\begin{tabular}{cccc}
\hline Eigenvalue & $\begin{array}{c}\text { Ability to clean up } \\
\text { space debris }\end{array}$ & Estimated price & Removal efficiency \\
\hline$\lambda_{\max }$ & 10.9326 & 10.2978 & 10.3760 \\
\hline
\end{tabular}

We can also calculate the corresponding eigenvectors.

$W^{(3)}=\left[\begin{array}{cccccccccc}0.0557 & 0.3702 & 0.4313 & 0.2266 & 0.4565 & 0.5117 & 0.2038 & 0.2010 & 0.0893 & 0.2497 \\ 0.0793 & 0.0471 & 0.3172 & 0.2751 & 0.0451 & 0.2905 & 0.2804 & 0.1470 & 0.1558 & 0.7774 \\ 0.0499 & 0.3634 & 0.2102 & 0.0537 & 0.3544 & 0.1969 & 0.0534 & 0.6511 & 0.4216 & 0.2226\end{array}\right]$

They are all pass the consistency check.

STEP 3 Total sorts of the layers--the one-time inspection

Then following results are obtained.

$$
\begin{aligned}
W & =W^{(3)} \times W^{(2)} \\
& =\left[\begin{array}{llllllllll}
0.0785 & 0.4672 & 0.3923 & 0.1724 & 0.4869 & 0.4017 & 0.1656 & 0.6961 & 0.4478 & 0.4245
\end{array}\right]^{\prime}
\end{aligned}
$$




\begin{tabular}{|c|c|c|}
\hline Definition & Weight in AHP & Star-level \\
\hline$C_{1}$ & 0.0785 & 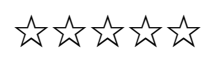 \\
\hline$C_{2}$ & 0.4672 & 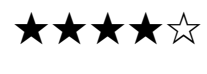 \\
\hline$C_{3}$ & 0.3923 & 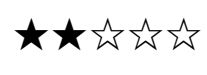 \\
\hline$C_{4}$ & 0.1724 & 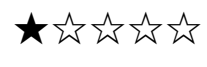 \\
\hline$C_{5}$ & 0.4869 & 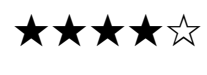 \\
\hline$C_{6}$ & 0.4017 & 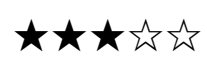 \\
\hline$C_{7}$ & 0.1656 & 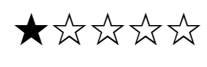 \\
\hline$C_{8}$ & 0.6961 & 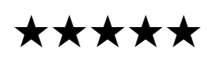 \\
\hline$C_{9}$ & 0.4478 & 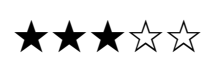 \\
\hline$C_{10}$ & 0.4245 & 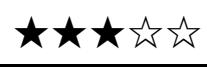 \\
\hline
\end{tabular}

We choose several main combinations in Table 3 for the next analysis, which is $C_{8}, C_{5}$ and $C_{2}$ according to importance of the weight.

\section{Conclusion}

Through the AHP model, we can find that the best way to remove the space debris is $C_{8}$, which stand for the way of $S G T+S S$. Finally, in this paper, through the establishment of the AHP model, we analyze the different combinations, and get the conclusion of the top three. These methods which we have chosen can make automatic space debris removal device having a better use value and market competitiveness.

\section{References}

[1]Hou L, Cai Y, Liu J, et al. Variable fidelity robust optimization of pulsed laser orbital debris removal under epistemic uncertainty[J]. Advances in Space Research, 2015.

[2]Vance L, Mense A. Value analysis for orbital debris removal[J]. Advances in Space Research, 2013, 52(4): 685-695.

[3] Phipps C R, Baker K L, Libby S B, et al. Removing orbital debris with lasers[J]. Advances in Space Research, 2012, 49(9): 1283-1300.

[4]Gong Songbo. Establishment and application of space debris evolution model [D]. Harbin Institute of Technology, 2005 